(Ref. 3) and is straightforward to verify.

${ }^{12}$ Yao has demonstrated the infrared finiteness of quark-quark scattering to lowest order (one-loop corrections to the elastic process and up to one undetected soft gluon in the final state) (Y. P. Yao, private com- munication).

${ }^{13} \mathrm{~J}$. M. Cornwall and G. Tiktopoulos, Phys. Rev. Lett. 35, 338 (1975), and University of California at Los Angeles Report No. UCLA/75/TEP/21, 1975, (to be published).

\title{
Hadron Multiplicities and Structure Functions-A Comparison of Muoproduction with Photoproduction and $e^{+} e^{-}$Annihilation*
}

\author{
K. Bunnell, M. Duong-van, R. Mozley, A. Odian, F. Villa, and L. C. Wang \\ Stanford Linear Accelevator Center, Stanford, California 94305 \\ and \\ C. del Papa, D. Dorfan, S. M. F. Flatté, C. A. Heusch, B. Lieberman, G. Luxton, † H. Meyer, ఫ \\ L. Moss, T. Schalk, and A. Seiden \\ University of California, Santa Cruz, California 95064 \\ (Received 3 February 1976)
}

\begin{abstract}
We report on mean hadron multiplicites and structure functions observed in muon-proton collisions at $14 \mathrm{GeV} / c$. A remarkable agreement is found with photoproduction and $e^{+} e^{-}$annihilation data.
\end{abstract}

Much interest has recently centered on photonhadron couplings in various domains. Meson photoproduction, initiated by lightlike photons, gave substance to the ideas of vector meson dominance. Deeply inelastic electroproduction established spacelike photons as particularly appropriate probes of nucleon structure at small distances, and made their hadronic couplings a matter of prime concern. $e^{+} e^{-}$annihilation proved to be a unique vehicle for an investigation of higher-mass mesonic systems coupling to timelike photons.

A direct comparison of global hadronic features in inelastic $e p$ or $\mu p$ cross sections with photoproduction and $e^{+} e^{-}$annihilation data may shed light on the underlying mechanisms. We therefore report on results we obtained in inelastic muon-proton scattering, ${ }^{1}$ and compare mean secondary hadron multiplicities and hadron structure functions with data recently obtained in photoproduction and in $e^{+} e^{-}$collisions.

Our data were obtained from the scattering of 14-GeV positive muons, ${ }^{2}$ in a new Stanford Linear Accelerator Center beam line, off liquid hydrogen inside a specially designed 2-m-long streamer chamber. ${ }^{3}$ This permitted essentially $4 \pi-\mathrm{sr}$ final-state charged-particle detection. The trigger was optimized for muon energy losses $\nu>2$ $\mathrm{GeV}$, and lepton-hadron momentum transfers ("photon mass"') $\left|-q^{2}\right|>0.3(\mathrm{GeV} / c)^{2}$. Some 8000 inelastic events were obtained.

Geometric losses turned out to be small and readily correctable, since charge conservation constrains the final-state hadrons to reflect a net charge of +1 . We describe our results in terms of the variables

$$
\begin{aligned}
& s=W^{2}=2 M \nu+M^{2}-Q^{2}, \\
& 1 / \omega=-q^{2} / 2 p \circ q=Q^{2} / 2 M \nu,
\end{aligned}
$$

where $M=$ proton mass, $Q^{2}=-q^{2}$, and $\nu$ is the photon energy in the lab system.

Figure 1(a) shows the mean multiplicities of charged hadrons plotted versus $s$ for $0.5 \leqslant Q^{2}$ $\leqslant 4.5$. In this range, where there is no significant $Q^{2}$ dependence, ${ }^{1}$ we observe a monotonic increase with $s$. A comparison with photoproduction $\left(Q^{2}=0\right)^{4}$ data shows some systematic differences mainly at the low- $W$ end. For a comparison with hadron multiplicities emerging from $e^{+} e^{-}$collisions,${ }^{5}$ we have to make allowances for the different charges $(Q, B, L)$ of the incoming channels, which constrain the distribution of the available energy onto final-state hadrons. We do this in two ways: (a) We leave $s=W^{2}$ as the energy available, but remove all one-charged-prong events from the $\mu p$ sample. This is approximately justified because events where the proton is the the only charged hadron in the final state are due largely to fragmentation of the spacelike photon into an all-neutral system; the corresponding process for timelike photons, $e^{+} e^{-} \rightarrow$ neutrals, is presently not easy to detect, and is not included in the quoted values of $\langle n\rangle_{e^{+} e^{-}}$. Moreover, the fragmentation of the $C$-odd photon into $C$-even 


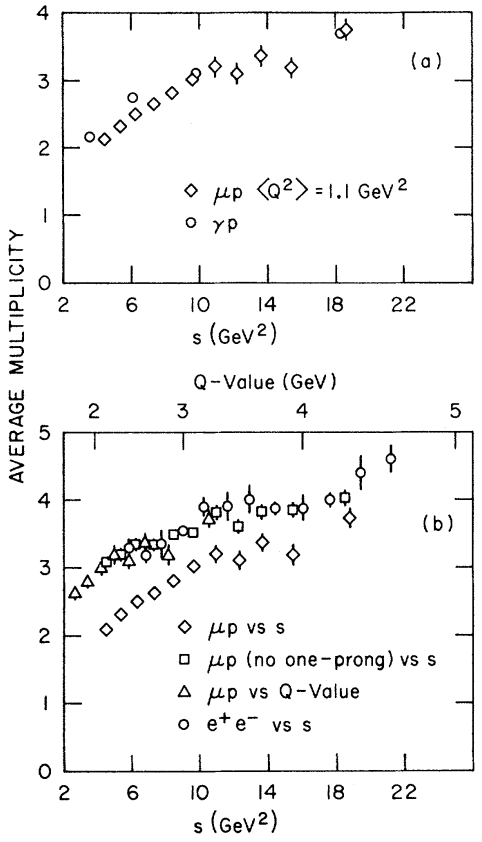

FIG. 1. (a) Comparison of the average hadronic charged-particle multiplicites $\langle\boldsymbol{n}\rangle$, as observed in photoproduction $\left(Q^{2}=0\right)$ and leptoproduction $\left(0.5 \leqslant Q^{2} \leqslant 4.5\right)$. (b) $\langle n\rangle$ comparison for $e^{+} e^{-}$annihilation and leptoproduction. As alternative methods of taking into account the different quantum number constraints in the two reactions, the leptoproduction data are also plotted with one-prong events deleted and versus the $Q$ value (defined in text) of the reaction.

states, such as $\pi^{0}, \eta^{0}, \ldots$, in the absence of a recoiling system is forbidden by $C$ invariance. (b) We choose as the appropriate energy variable the so-called $Q$ value (unrelated to $Q^{2}$ above)

$$
Q \text { value }=s^{1 / 2}-\left(m_{1}+m_{2}\right),
$$

which is a measure for the energy available for hadron formation in the interaction. We somewhat arbitrarily set $m_{1}+m_{2}=m_{p}+m_{\pi}$ to reflect the minimum rest mass that can be adopted by the final state. For $e^{+} e^{-} \rightarrow$ hadrons, $Q$ value $\approx \sqrt{s}$ to good precision.

Figure 1(b) shows, for both cases (a) (squares) and (b) (triangles, upper scale), that the comparison of $\mu p$ and $e^{+} e^{-}$data is good within the experimental uncertainties. It is also clear that a straightforward comparison for the full $\mu p$ sample (diamonds) at comparable $s$ disagrees strongly at low energies.

We now turn to an examination of the hadron "structure functions," which describe for both interactions how they distribute the energy available among the secondary hadrons. Using the

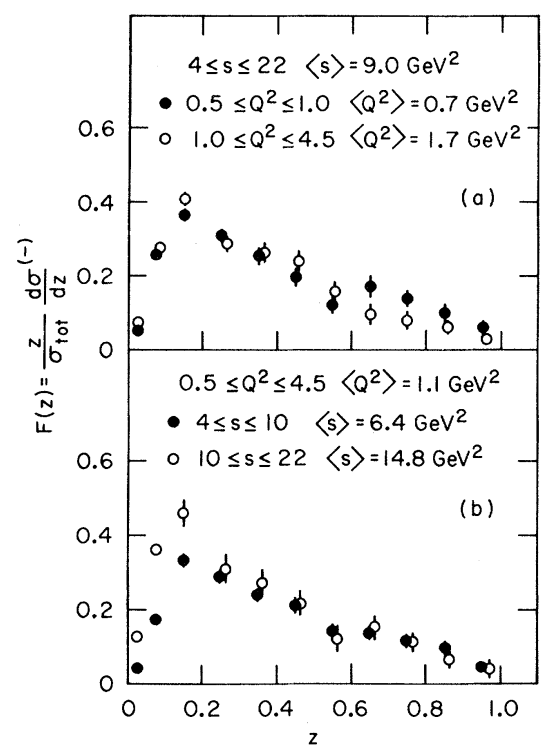

FIG. 2. (a) Leptoproduction "structure function" for the production of negative hadrons, plotted for two $Q^{2}$ intervals. (b) Same quantity plotted for two $s$ intervals.

\section{fractional energy variables}

$$
\begin{aligned}
& z=E_{h}{ }^{1 \mathrm{ab}} / \nu \text { for } \mu p \rightarrow \mu+\text { hadrons, } \\
& x=2 E_{h} / \sqrt{s} \text { for } e^{+} e^{-} \rightarrow \text { hadrons, }
\end{aligned}
$$

and the single-hadron inclusive cross sections, we define these functions by the invariant quantities $^{6}$

$$
\begin{aligned}
& \boldsymbol{F}_{\mu p}^{(-)}=\left(z / \sigma_{\text {tot }}\right) d \sigma^{(-)} / d z, \\
& F_{e^{+} e^{-}}=\left(x / 4 \sigma_{\text {tot }}\right) d \sigma / d x .
\end{aligned}
$$

$F_{\mu p}$ is restricted here to negative hadrons to avoid $\pi^{+}$-proton confusion, since the streamer chamber did not provide particle identification. Note that $z$ normalizes the lab hadron energy with respect to the photon energy alone, just as the $Q$-value definition subtracts rest masses from the energy $W$.

Figure 2(a) shows the structure function $F_{\mu p}{ }^{(-)}$ for two $Q^{2}$ intervals. A $Q^{2}$ dependence, if at all, may be showing up at $z$ values $>0.5$. A breakdown into two $s$ intervals, after integration over $Q^{2}$ [Fig. 2(b)], shows an increase with energy at small $z$ values only. Note that small $z$ corresponds to the central and proton fragmentation regions. The area under the curves is, in the lab system, the fraction of the energy carried by negative particles (which we assume to be pions). For fractional hadron energies above $z=0.4$, $F_{\mu p}^{(-)}$is approximately energy independent, and therefore exhibits scaling behavior. 


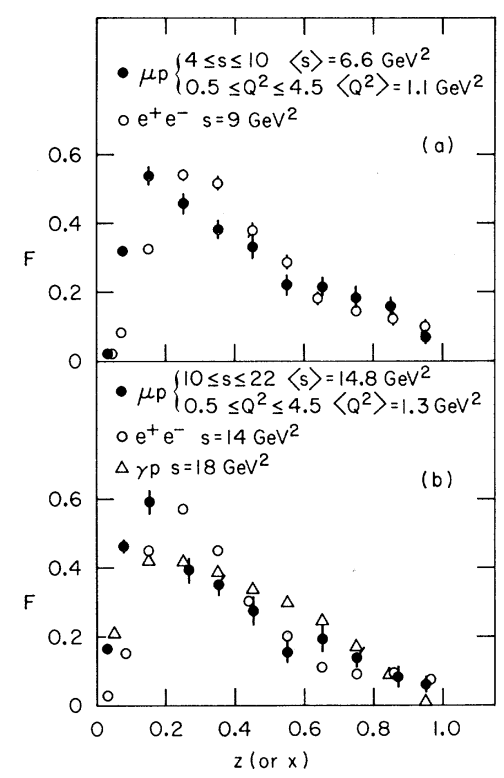

FIG. 3. (a) Comparison of leptoproduction and $e^{+} e^{-}$ annihilation "structure functions" for small $s$. (b) Same quantities at larger $s$. Also shown is the analogous function for photoproduction.

To compare with $e^{+} e^{-}$annihilation data, we again eliminate "one-prong" events, this time from the normalizing $\sigma_{\text {tot }}$. In Figs. $3(\mathrm{a})$ and (b), we show the corresponding function $F_{\mu p}^{(-))}=(z /$ $\left.\sigma^{-}\right) d \sigma^{(-)} / d z$ [where $\sigma^{-} \equiv \sigma_{\text {tot }}-\sigma$ (one-prong) $]$ for our $\mu p$ data along with $F_{e^{+}} e^{-}$. In the definition of $F_{e^{+} e^{-}}$, the factor $\frac{1}{4}$, needed to make the comparisons, is due to two effects, each contributing a factor of $\frac{1}{2}$. The first of these is due to the inclusion of both hadron charges as opposed to negatives only for $F_{\mu p}^{(-)}$; the second stems from the fact that timelike photons fragment into two leading quarks versus one leading quark for spacelike photons. With this normalization, the area under the function $\left(x / 4 \sigma_{\text {tot }}\right) d \sigma / d x$ measures the fraction of the energy carried by negatives produced in $e^{+} e^{-}$collisions, analogous to $F_{\mu p}^{(-)}$.

The comparison shows a close similarity in overall shape (note that the normalization determines the areas under the two sets of curves). Above $z$ (or $x$ ) values of $\sim 0.4$, the pion structure functions for deeply inelastic scattering and for $e^{+} e^{-}$annihilation agree within errors. The data in Fig. 2(b) show that this agreement is equally good if we normalize $F_{\mu p}$ to $\sigma_{\text {tot }}$. At small $z$ values, $0.1 \lessgtr z \lesssim 0.2$, "proton fragmentation," which is absent in the $e^{+} e^{-}$annihilation process (bar- yon number $=0$ ) appears to add to the structure function for the spacelike photon case. In the quark-parton picture, the $F$ functions for large arguments $z$ or $x$ represent "leading quark" fragmentation. Our comparison is valid at the energies presented here, since the quark mixtures from which the leading quark is chosen favor the charge $+\frac{2}{3} p$-type quark in both cases.

We also compare with the lightlike photon case, where we identify $z$ as the laboratory variable $z$ $=E_{h} / E_{\gamma}$. The triangles in Fig. 3(b) represent photoproduction data ${ }^{7}$ at $s \approx 20$; to allow a meaningful comparison of real-photon fragmentation with the spacelike and timelike cases, the (sizable) diffractive part was subtracted to a good approximation by removal of all $\rho^{0}$ photoproduction events. The data are seen to be consistent within the errors of this approximation, for $z$ $>0.4$.

We conclude that the global features of photon fragmentation are remarkably universal in all kinematical regimes, at $s$ values between 5 and $20 \mathrm{GeV}^{2}$ : For appropriately defined data samples, our muoproduction results on charged hadron multiplicities and $\boldsymbol{F}$ functions (often referred to as "structure functions") yield results closely similar to photoproduction and $e^{+} e^{-}$annihilation data. It will be interesting to reach an understanding of differences in the detailed structure, mainly at small $z$ values.

*Work supported in part by the U. S. Energy Research and Development Administration.

$\dagger$ Now at Stanford Medical Center, Stanford University, Stanford, Calif. 94305.

\$Visitor from DESY, Deutsches Elektronen-Synchrotron, Hamburg, Germany.

${ }^{1}$ C. del Papa et al., Phys. Rev. D (to be published).

${ }^{2}$ S. Flatté, C. A. Heusch, and A. Seiden, Nucl. Instrum. Methods 119,333 (1974)。

${ }^{3}$ C. A. Heusch, B. Lieberman, and A. Seiden, Nucl. Instrum. Methods 124, 165 (1975).

${ }^{4} \mathrm{~J}$. Ballam et al., Phys. Rev. D 5 , 545 (1972); H. H. Bingham et al., Phys. Rev. D $\underline{8}, 1277$ (1973).

${ }^{5}$ M. L. Perl, private communication.

${ }^{6}$ S. D. Drell and T. M. Yan, Phys. Rev. Lett. 24, 855 (1970); E. W. Colglazier and F. Ravndal, Phys. Rev. D 7,1537 (1973). For another use of this approach see J. T. Dakin and G. J. Feldman, Phys. Rev. D $\underline{8}$, 2862 (1973).

${ }^{7}$ We thank Dr. Eliezer Kogan for making the photoproduction comparison data available to us. 